\title{
Enhanced Biot's Finite Element Displacement Formulation for Porous Materials and Original Resolution Methods Based on Normal Modes
}

\author{
Olivier Dazel, Bruno Brouard, Nicolas Dauchez, Alan Geslain \\ Laboratoire d'Acoustique de l'Université du Maine - UMR CNRS 6613, Avenue Olivier Messiaen, \\ 72085 Le Mans Cedex, France. olivier.dazel@univ-lemans.fr
}

\begin{abstract}
Summary
The use of finite element modeling for porous sound absorbing materials is often limited by the numerical cost of the resolution scheme. To overcome this limitation, an alternative finite element formulation for poroelastic materials modelled with the Biot-Allard theory is first presented. This formulation is based on the solid and total displacement fields of the porous medium. Three resolution methods (one semi-analytical and two numerical) based on normal modes are proposed secondly. These methods take benefit from the decoupling properties of normal modes. The semi-analytical method is associated with problems in which the shear wave can be neglected. The numerical methods are a direct and an iterative scheme. The direct method allows a reduction by 2 of the number of degrees without making any approximation. The iterative method provides an approximation corresponding to a controlled tolerance. The finite element formulation is validated by comparison with an analytical model in two mono-dimensional configurations corresponding to a single and a multilayered problem. The efficiency of the two numerical resolution methods is also illustrated in term of computation time in comparison with classical formulations, such as the mixed displacement-pressure formulation.
\end{abstract}

PACS no. 43.20.Bi

\section{Introduction}

Porous structures are used in automotive, aeronautics, building industries as passive absorbers in order to reduce noise annoyance. The modeling of the physics of these materials has been the subject of many scientific works in the last two decades and many models were proposed. Among them, Biot-Allard's [1, 2, 3, 4, 5, 6] theory of wave propagation in sound absorbing material is now considered as the reference. Even if this theory could still be enhanced, it is now common for the community that it is well fitted for the modeling of sound absorbing materials used in classical acoustical applications. This theory has nevertheless two main drawbacks. The first one is the difficulty of characterizing some of its intrinsic parameters, especially (visco-)elastic coefficients [7]. The second limitation is linked to the tremendous calculations induced by the resolution of these equations when the behaviour of the porous material cannot be predicted with classical plane waves techniques or analytical methods. This paper is concerned with the second drawback.

In Biot-Allard's theory, the porous medium is assumed as an aggregate, superposition of a solid and a fluid phase. Different formulation of Biot's theory have been proposed

Received 19 September 2008 ,

accepted 27 January 2009 in the literature and they can be grouped in two sets. The first one is related to mixed formulation $[8,9]$ and considers one displacement field and the interstitial pressure in the fluid. The formulation by Atalla et al. [8, 9] involves the full Biot-Allard model and the paper from Bermudez et al. [10] is a rigorous mathematical study for a non dissipative (and physically not realistic for acoustical applications) porous medium. The main drawback of Atalla et al. approach is to be only valid for harmonic motions [8] (due to the frequential dependance of JohnsonAllard model $[3,4,5])$. The second set of methods is related to displacement formulations and involves two displacement fields. The displacement of the solid phase can be aggregated with the one of the fluid phase [1] $u^{f}$ or with relative flow [2] between the solid and fluid phase $w=\phi\left(u^{f}-u^{s}\right)$ or, more recently with total displacement [11] $u^{t}=(1-\phi) u^{s}+\phi u^{f} . \phi$ denotes the porosity.

From a numerical point of view, mixed formulations have the advantage that finite element discretization leads to problems with four degrees of freedom (dof) per node instead (and an additional node per element to avoid instability and wrong numerical applications in certain cases) of six for 3D problems. Their drawbacks are an intricate physical interpretation and conditioning problems. In addition, they are only valid for harmonic problems and they need five elementary matrices (two for each phase and one corresponding to coupling) instead of three for dis- 
placement formulations. In order to reduce computation time, the choice of mixed formulations has been preferred during the last decade and two main sets of improvement methods have nevertheless been proposed in the literature. The first one consists in improving the quality of the finiteelement process by using hierarchical elements [12, 13]. The second one is to use modal techniques. One can cite decoupled modal analysis [14] and generalized complex modes techniques $[15,16]$. The first modal technique is shown not efficient enough and the second one presents a mathematical formalism and ill conditioned computation techniques which are difficult to apply for non-academic problems.

The main idea of this paper is to propose a new approach to solve numerical poroelastic problems inspired from the resolution of analytical methods. Three resolution methods (one semi-analytical and two numerical) based on normal modes are proposed. These methods take benefit from the decoupling properties of normal modes. The semi-analytical method is associated with problems in which the shear wave can be neglected (in the case of monodimensional problems or when the material is noncohesive [17]). Even if the solving method is analytical, it is called semi-analytical as a numerical finite-element problem is concerned. The numerical methods are a direct and an iterative scheme. The iterative method provides an approximation corresponding to a controlled tolerance. The finite element formulation is validated by comparison with an analytical model in 2 mono-dimensional configurations corresponding to a single and a multilayered problem. The efficiency of the two numerical resolution methods is also illustrated in terms of computation time in comparison with classical formulations, such as the mixed displacement-pressure formulation. In addition, from the knowledge of the authors, the proposed resolution methods can not be applied to mixed formulation finite-element problems due to the coupling between displacement and pressure modes.

Section 2 presents the $\left\{\mathbf{u}^{s}, \mathbf{u}^{t}\right\}$ finite-element formulation. Two numerical methods for the resolution of the numerical system are proposed in section 3. The compressional case is presented in section 4. The proposed approach is compared to the classical formulation of the literature in section 5. Section 6 concludes the paper.

\section{2. $\left\{\mathbf{u}^{s}, \mathbf{u}^{t}\right\}$ Finite element formulation}

\section{1. $\left\{\mathbf{u}^{s}, \mathbf{u}^{t}\right\}$ Equations of Biot's theory}

The $\left\{\mathbf{u}^{s}, \mathbf{u}^{t}\right\}$ representation of Biot's theory has been proposed by Dazel et al. [11]. It is an equivalent formulation to the $\left\{\mathbf{u}^{s}, \mathbf{u}^{f}\right\}$ one which has the advantage of simplifying stress-strain relations together with coupling conditions. The motion equations are given by:

$$
\begin{aligned}
\nabla \cdot \hat{\boldsymbol{\sigma}}\left(u^{s}\right) & =-\omega^{2} \widetilde{\rho}_{s} u^{s}-\omega^{2} \widetilde{\rho}_{e q} \tilde{\gamma} u^{t}, \\
\widetilde{K}_{e q} \nabla \nabla \cdot u^{t} & =-\omega^{2} \widetilde{\rho}_{e q} \tilde{\gamma} u^{s}-\omega^{2} \widetilde{\rho}_{e q} u^{t} .
\end{aligned}
$$

$u^{s}$ and $u^{t}$ are solid and total displacement fields, $\widetilde{K}_{e q}$ corresponds to the equivalent compressibility of the fluid, $\tilde{\rho}_{e q}$ and $\tilde{\rho}_{s}$ are equivalent densities, $\tilde{\gamma}$ is a coupling coefficient and $\hat{\sigma}$ is the in-vacuo stress tensor of the solid phase. The motion equations (1) are associated to the following constitutive relations:

$$
\begin{aligned}
\hat{\sigma}_{i j}\left(u^{s}\right) & =\hat{A} \nabla \cdot u^{s} \delta_{i j}+N\left(\frac{\partial u_{i}^{s}}{\partial x_{j}}+\frac{\partial u_{j}^{s}}{\partial x_{i}}\right), \\
P & =-\widetilde{K}_{e q} \nabla \cdot u^{t} .
\end{aligned}
$$

$P$ is the interstitial pressure in the porous medium. All the coefficient of these equations can be obtained with Biot-Allard's theory and their expression are given in Appendix A1.

In addition, this formulation simplifies the continuity relations at the interface between porous structure and another porous or a fluid structure. The continuity relations [11] at the interface between two porous media are simply the continuity of solid and normal total displacements as well as the continuity of in-vacuo stress tensor and pressure. In the case of an interface between a porous material and a fluid, one has the equality between the pressure of the fluid and the pressure of the porous medium as well as the equality between the normal total displacement and the normal displacement of fluid and the nullity of the invacuo stress.

\subsection{Variational formulation and discretized form}

The finite element formulation [18] of the $\left\{\mathbf{u}^{s}, \mathbf{u}^{t}\right\}$ formulation is now proposed. The method to discretize a poroelastic variational formulation has been proposed by various authors $[19,13,8]$. Let $\Omega$ be the porous material domain and $\partial \Omega$ its boundary. This boundary can be divided in two parts $\partial \Omega_{D}$ and $\partial \Omega_{N}$. The methodology to obtain the variational formulation is in three steps. The first one is the multiplication of equations (1a) and (1b) by variation fields $\delta u^{s}$ and $\delta u^{t}$. These fields are chosen in order to be kinematically admissible (i.e. to satisfy the homogeneous Dirichlet boundary conditions on $\partial \Omega_{D}$ ). The two equations are then integrated over $\Omega$ and a Green formula is applied to the stress terms. The variational form associated to solid and fluid phase are then

$$
\begin{aligned}
& \int_{\Omega} \hat{\boldsymbol{\sigma}}\left(u^{s}\right): \varepsilon\left(\delta u^{s}\right)-\omega^{2}\left(\tilde{\rho}_{s} u^{s}+\widetilde{\gamma} \tilde{\rho}_{e q} u^{t}\right) \delta u^{s} \mathrm{~d} \Omega \\
& =\oint_{\partial \Omega_{N}} \hat{\boldsymbol{\sigma}}\left(u^{s}\right) \cdot \mathbf{n} \delta u^{s} \mathrm{~d} \Gamma, \\
& \int_{\Omega} \widetilde{K}_{e q} \nabla \cdot u^{t} \nabla \cdot \delta u^{t}-\omega^{2} \widetilde{\rho}_{e q}\left(u^{s}+\tilde{\gamma} u^{t}\right) \delta u^{t} \mathrm{~d} \Omega \\
& =\oint_{\partial \Omega_{N}} \tilde{K}_{e q} \nabla \cdot u^{t}\left(\delta u^{t} \cdot \mathbf{n}\right) \mathrm{d} \Gamma .
\end{aligned}
$$

$\mathbf{n}$ is the outwarding normal vector at the boundary. The boundary integral on $\partial \Omega_{D}$ is null due to Dirichlet conditions. These formulations can then be discretized by finite element. The porous domain $\Omega$ is first partitioned in $n$ elementary sub-domains $\Omega_{e}$. On each sub-domain, a finite 
number of nodes are chosen as well as interpolation functions. The solid and total displacement fields can then be approached on element $e$ in terms of the nodal displacements approximations $\mathbf{u}_{e}^{s}$ and $\mathbf{u}_{e}^{t}$.

$$
\left\{\begin{array}{c}
u_{e}^{s} \\
u_{e}^{t}
\end{array}\right\} \approx\left[N_{u^{s}}^{e} \mid N_{u^{t}}^{e}\right]\left\{\begin{array}{l}
\mathbf{u}_{e}^{s} \\
\mathbf{u}_{e}^{t}
\end{array}\right\}
$$

where $\left[N_{u^{s}}^{e}\right]\left(\right.$ resp. $\left.\left[N_{u^{t}}^{e}\right]\right)$ corresponds to the shape function associated to the solid (resp. total) displacement. In all the following, the interpolation functions of the solid and total displacement are assumed equals (and denoted by $\left.\left[N_{u}\right]\right)$. Three elementary matrices are defined on element $e$ :

$$
\begin{aligned}
{\left[\mathbf{M}_{0}^{e}\right] } & =\int_{\Omega_{e}}\left[N_{u}^{e}\right]^{t}\left[N_{u}^{e}\right] \mathrm{d} \Omega, \\
{\left[\mathbf{K}_{i}^{e}\right] } & =\int_{\Omega_{e}}\left[B_{u}^{e}\right]^{t}\left[D_{i}\right]\left[B_{u}^{e}\right] \mathrm{d} \Omega, \quad i=0,1
\end{aligned}
$$

$\left[\mathbf{M}_{0}^{e}\right]$ is associated with the $\mathbf{u} \cdot \delta \mathbf{u}$ terms of the variational formulations. $\left[\mathbf{K}_{0}^{e}\right]$ is associated with the $\nabla \cdot u \nabla \cdot \delta u$ products and $\left[\mathbf{K}_{1}^{e}\right]$ corresponds to the shear terms. Some details on the elementary matrices can be found in Appendix A2.1. Two elementary vectors are also defined. On $\partial \Omega_{D}$, the normal stresses are imposed and thereby known. The elementary force vectors are:

$$
\begin{aligned}
{\left[\mathbf{F}_{s}^{e}\right] } & =\oint_{\partial \Omega_{e}}\left[N_{u}^{e}\right]^{t} \hat{\boldsymbol{\sigma}}\left(u^{s}\right) \mathrm{d} \Gamma, \\
{\left[\mathbf{F}_{t}^{e}\right] } & =\oint_{\partial \Omega_{e}}\left[N_{u}^{e}\right]^{t} \widetilde{K}_{e q} \nabla \cdot u^{t} \cdot \mathbf{n} \mathrm{d} \Gamma .
\end{aligned}
$$

$\partial \Omega_{e}$ corresponds to the surface of the element included in the boundary $\partial \Omega_{N}$. If an element is not linked to $\partial \Omega_{N}$, the elementary forces are null. The global mass and stiffness matrices and forcing vector are obtained by the summation of elementary matrices. Let $\mathbf{u}^{i}(i=s, t)$ be the global displacement vector corresponding to the global degree of freedom of the problem. The displacement unknowns in the element and in the global vector are linked through a boolean matrix $\left[\mathbf{L}_{e}\right]$ so that $\mathbf{u}_{e}^{i}=\left[\mathbf{L}_{e}\right] \mathbf{u}^{i}$. The global pseudo-mass and pseudo-stiffness matrices and forces then reads:

$$
\begin{aligned}
{\left[\mathbf{M}_{0}\right] } & =\sum_{e \subset \Omega}\left[\mathbf{L}_{e}\right]^{t}\left[\mathbf{M}_{0}^{e}\right]\left[\mathbf{L}_{e}\right] \\
{\left[\mathbf{K}_{i}\right] } & =\sum_{e \subset \Omega}\left[\mathbf{L}_{e}\right]^{t}\left[\mathbf{K}_{i}^{e}\right]\left[\mathbf{L}_{e}\right] \\
\mathbf{F}^{s, t} & =\sum_{e \subset \Omega}\left[\mathbf{L}_{e}\right]^{t}\left[\mathbf{F}_{s, t}^{e}\right]
\end{aligned}
$$

They are called pseudo as they are not respectively homogeneous to mass and stiffness but it can be shown that they just differ by a multiplicative constant. The final linear system then reads:

$$
\begin{aligned}
& \left(\left[\begin{array}{cc}
\hat{P}\left[\mathbf{K}_{0}\right]+N\left[\mathbf{K}_{1}\right] & {[0]} \\
{[0]} & \widetilde{K}_{e q}\left[\mathbf{K}_{0}\right]
\end{array}\right]\right. \\
& \left.-\omega^{2}\left[\begin{array}{cc}
\tilde{\rho}_{s}\left[\mathbf{M}_{0}\right] & \tilde{\gamma} \widetilde{\rho}_{e q}\left[\mathbf{M}_{0}\right] \\
\tilde{\gamma} \tilde{\rho}_{e q}\left[\mathbf{M}_{0}\right] & \tilde{\rho}_{e q}\left[\mathbf{M}_{0}\right]
\end{array}\right]\right)\left\{\begin{array}{l}
\mathbf{u}^{s} \\
\mathbf{u}^{t}
\end{array}\right\}=\left\{\begin{array}{l}
\mathbf{F}^{s} \\
\mathbf{F}^{t}
\end{array}\right\} .
\end{aligned}
$$

$\mathbf{u}^{s}$ and $\mathbf{u}^{t}$ are both of size $n$. This system is of size $2 n$ and mathematically equivalent to the one obtained by the discretization of either $\left\{\mathbf{u}^{s}, \mathbf{u}^{f}\right\}$ or $\left\{\mathbf{u}^{s}, \mathbf{w}\right\}$ formulations of Biot's theory. It can be checked by making the adequate linear combinations but it is a consequence of the equivalence of the three formulations for continuous problems. By comparison to these two formulations, this system has the advantage to provide a diagonal by block stiffness matrix. This property is now used in order to solve this system more efficiently.

\section{Proposed numerical approaches of reso- lution based on normal modes}

\subsection{Introduction}

In this section, two numerical methods of resolution are proposed. They are based on normal modes. The first one is a direct (exact) method of resolution on a problem of size $n$ (and not $2 n$ as in the initial problem, equation 8 ). The second method is an iterative one based on Jacobi method.

The definition of normal modes and their properties are now briefly recalled. Let $\left[\boldsymbol{\Phi}_{0}\right]$ (resp. $\left[\boldsymbol{\Phi}_{1}\right]$ ) and $\left[\mathbf{k}_{0}^{2}\right]$ (resp. $\left.\left[\mathbf{k}_{1}^{2}\right]\right)$ be the eigenvectors and the diagonal matrix of eigenvalues of the generalized problem $\left[\mathbf{K}_{0}\right]-\omega^{2}\left[\mathbf{M}_{0}\right]$ (resp. $\left.\left[\mathbf{K}_{0}+(N / \hat{P}) \mathbf{K}_{1}\right]-\omega^{2}\left[\mathbf{M}_{0}\right]\right)$. It can be shown that the eigenvalues are homogeneous to a square of wave number and note that $N / \hat{P}$ is real. Each basis can be chosen so that the modes are normalized with respect to the $\left[\mathbf{M}_{0}\right]$ matrix,

$$
\begin{aligned}
{\left[\mathbf{\Phi}_{0}\right]^{t}\left[\mathbf{K}_{0}\right]\left[\mathbf{\Phi}_{0}\right] } & =\left[\mathbf{k}_{0}^{2}\right], \\
{\left[\mathbf{\Phi}_{1}\right]^{t}\left[\mathbf{K}_{0}+\frac{N}{\hat{P}} \mathbf{K}_{1}\right]\left[\mathbf{\Phi}_{1}\right] } & =\left[\mathbf{k}_{1}^{2}\right], \\
{\left[\mathbf{\Phi}_{i}\right]^{t}\left[\mathbf{M}_{0}\right]\left[\mathbf{\Phi}_{i}\right] } & =[\mathbf{I}], \quad i=0,1 .
\end{aligned}
$$

I is the identity matrix of size $n$. These last relations are called orthogonality relations and involves the decoupling of modes. This decoupling is nevertheless not preserved while considering the coupled projection. One then defined

and

$$
\begin{aligned}
{\left[\mathbf{C}_{1}\right] } & =\left[\mathbf{\Phi}_{0}\right]^{t}\left[\mathbf{K}_{1}\right]\left[\mathbf{\Phi}_{0}\right] \\
{\left[\mathbf{C}_{2}\right] } & =\left[\boldsymbol{\Phi}_{0}\right]^{t}\left[\mathbf{M}_{0}\right]\left[\boldsymbol{\Phi}_{1}\right] .
\end{aligned}
$$

In general, these two matrices are not diagonal nor even sparse. It is the main drawback of this method.

\subsection{Direct resolution}

The first numerical method only involves the compressional normal modes: one express the total and fluid displacement by the way of their modal contributions $\mathbf{q}^{s}$ and $\mathbf{q}^{t}$ on the $\left[\boldsymbol{\Phi}_{0}\right]$ basis. One then has:

$$
\begin{aligned}
\left\{\begin{array}{l}
\mathbf{u}^{s} \\
\mathbf{u}^{t}
\end{array}\right\} & =\left[\begin{array}{cc}
{\left[\mathbf{\Phi}_{0}\right]} & {[\mathbf{0}]} \\
{[\mathbf{0}]} & {\left[\boldsymbol{\Phi}_{0}\right]}
\end{array}\right]\left\{\begin{array}{l}
\mathbf{q}^{s} \\
\mathbf{q}^{t}
\end{array}\right\}, \\
\left\{\begin{array}{l}
\mathbf{F}^{\prime s} \\
\mathbf{F}^{\prime t}
\end{array}\right\} & =\left[\begin{array}{cc}
{\left[\boldsymbol{\Phi}_{0}\right]} & {[\mathbf{0}]} \\
{[\mathbf{0}]} & {\left[\boldsymbol{\Phi}_{0}\right]}
\end{array}\right]\left\{\begin{array}{l}
\mathbf{F}^{s} \\
\mathbf{F}^{t}
\end{array}\right\} .
\end{aligned}
$$


The projection of ( 8$)$ then reads

$$
\begin{aligned}
& \left(\begin{array}{cc}
{\left[\hat{P}\left[\mathbf{k}_{0}^{2}\right]+N\left[\mathbf{C}_{1}\right]\right.} & {[\mathbf{0}]} \\
{[\mathbf{0}]} & \widetilde{K}_{e q}\left[\mathbf{k}_{0}^{2}\right]
\end{array}\right] \\
& \left.-\omega^{2}\left[\begin{array}{cc}
\tilde{\rho}_{s}[\mathbf{I}] & \tilde{\gamma} \tilde{\rho}_{e q}[\mathbf{I}] \\
\tilde{\gamma} \tilde{\rho}_{e q}[\mathbf{I}] & \tilde{\rho}_{e q}[\mathbf{I}]
\end{array}\right]\right)\left\{\begin{array}{l}
\mathbf{q}^{s} \\
\mathbf{q}^{t}
\end{array}\right\}=\left\{\begin{array}{l}
\mathbf{F}^{s} \\
\mathbf{F}^{\prime t}
\end{array}\right\} .
\end{aligned}
$$

It is now straightforward that the second half of the linear system only involves diagonal matrices. It can then be easily inverted, thereby allowing a simple relation between $\mathbf{q}^{s}$ and $\mathbf{q}^{t}$,

$$
\mathbf{q}^{t}=\tilde{\gamma}\left[\mathbf{R}_{e q}\right] \mathbf{q}^{s}+\left[\mathbf{R}_{e q}\right] \frac{\mathbf{F}^{\prime t}}{\omega^{2} \widetilde{\rho}_{e q}}
$$

with the diagonal matrix $\left[\mathbf{R}_{e q}\right]$ defined by

$$
\left[\mathbf{R}_{e q}\right]=\operatorname{diag}\left(\frac{\delta_{e q}^{2}}{k_{i}^{2}-\delta_{e q}^{2}}\right), \quad \delta_{e q}^{2}=\frac{\omega^{2} \widetilde{\rho}_{e q}}{\widetilde{K}_{e q}}
$$

$k_{i}$ denotes the elements of $\left[\mathbf{k}_{0}\right]$ and $\delta_{e q}$ is the wave number of the equivalent fluid model wave. The expression of $\mathbf{q}^{t}$ can then be reintroduced in the first half of the system and one has

$$
\begin{aligned}
\left(\hat{P}\left[\mathbf{k}_{0}^{2}\right]+N\left[\mathbf{C}_{1}\right]-\omega^{2}\left\{\tilde{\rho}_{s}\right.\right. & \left.\left.+\widetilde{\gamma}^{2} \widetilde{\rho}_{e q}\left[\mathbf{R}_{e q}\right]\right\}\right) \mathbf{q}^{s} \\
& =\mathbf{F}^{\prime s}+\tilde{\gamma}\left[\mathbf{R}_{e q}\right] \mathbf{F}^{\prime t} .
\end{aligned}
$$

This system is of size $n$. The modal decoupling property was then used to divide by 2 the size of the system. The problem (15) can now be solved by a direct solver to find the value of $\mathbf{q}^{s}$.

To summarize, this technique is performed in two steps. The first step is the modal calculation and the projection of the matrices to obtain $\left[\mathbf{C}_{1}\right]$. These two operations are performed on real matrices of size $n$, are just done once and do not need to be done at each frequency. The second step is the resolution of the complex system (15) of size $n$ at each frequency, the post-processing to obtain $\mathbf{q}^{t}$ through (13) and the unknown displacements with (11). This postprocessing is very low consuming compared to the resolution of the linear system. The classical resolution methods involves the resolution of a complex linear system of size $2 n$ at each frequency. The comparison of computational time of both methods is presented in section 5 .

\subsection{Iterative resolution}

A second numerical method is now proposed to solve system (8). Unlike the previous one, it is not a direct method but an iterative one. The nodal displacements are expressed in the $\left\{\left[\boldsymbol{\Phi}_{1}\right],\left[\boldsymbol{\Phi}_{0}\right]\right\}$ basis as

$$
\begin{gathered}
\left\{\begin{array}{l}
\mathbf{u}^{s} \\
\mathbf{u}^{t}
\end{array}\right\}=\left[\begin{array}{cc}
{\left[\mathbf{\Phi}_{1}\right]} & {[\mathbf{0}]} \\
{[\mathbf{0}]} & {\left[\mathbf{\Phi}_{0}\right]}
\end{array}\right]\left\{\begin{array}{l}
\left.\mathbf{q}^{s}\right\} \\
\mathbf{q}^{t}
\end{array}\right\}, \\
\left\{\begin{array}{l}
\mathbf{F}^{\prime s} \\
\mathbf{F}^{\prime t}
\end{array}\right\}=\left[\begin{array}{cc}
{\left[\boldsymbol{\Phi}_{1}\right]} & {[\mathbf{0}]} \\
{[\mathbf{0}]} & {\left[\boldsymbol{\Phi}_{0}\right]}
\end{array}\right]^{t}\left\{\begin{array}{l}
\mathbf{F}^{s} \\
\mathbf{F}^{t}
\end{array}\right\} .
\end{gathered}
$$

The projection of (8) then reads

$$
\begin{array}{r}
\left.\left(\begin{array}{cc}
\hat{P}\left[\mathbf{k}_{1}^{2}\right] & {[\mathbf{0}]} \\
{[\mathbf{0}]} & \widetilde{K}_{e q}\left[\mathbf{k}_{0}^{2}\right]
\end{array}\right]-\omega^{2}\left[\begin{array}{cc}
\tilde{\rho}_{s}[\mathbf{I}] & \tilde{\gamma} \tilde{\rho}_{e q}\left[\mathbf{C}_{2}\right] \\
\tilde{\gamma} \tilde{\rho}_{e q}\left[\mathbf{C}_{2}\right]^{t} & \tilde{\rho}_{e q}[\mathbf{I}]
\end{array}\right]\right) \\
\cdot\left\{\begin{array}{l}
\mathbf{q}^{s} \\
\mathbf{q}^{t}
\end{array}\right\}=\left\{\begin{array}{l}
\mathbf{F}^{\prime s} \\
\mathbf{F}^{t}
\end{array}\right\}
\end{array}
$$

The matrix of this problem can be written as the difference of two matrices $[\mathbf{M}]$ and $[\mathbf{N}]$ defined by:

$$
\begin{aligned}
& {[\mathbf{M}]=\left[\begin{array}{cc}
\hat{P}\left[\mathbf{k}_{1}^{2}\right]-\omega^{2} \widetilde{\rho}_{s}[\mathbf{I}] & {[\mathbf{0}]} \\
{[\mathbf{0}]} & \widetilde{K}_{e q}\left[\mathbf{k}_{0}^{2}\right]-\omega^{2} \widetilde{\rho}_{e q}[\mathbf{I}]
\end{array}\right],} \\
& {[\mathbf{N}]=\omega^{2} \widetilde{\gamma} \widetilde{\rho}_{e q}\left[\begin{array}{cc}
{[\mathbf{0}]} & {\left[\mathbf{C}_{2}\right]} \\
{\left[\mathbf{C}_{2}\right]^{t}} & {[\mathbf{0}]}
\end{array}\right] .}
\end{aligned}
$$

$[\mathbf{M}]$ is a diagonal matrix which is easy to invert. The iterative "porous-Jacobi" method consists in choosing an initial vector $\mathbf{X}_{0}$ and to iterate the relation

$$
\mathbf{X}_{i+1}=[\mathbf{M}]^{-1}\left([\mathbf{N}] \mathbf{X}_{i}+\left\{\begin{array}{l}
\mathbf{F}^{\prime s} \\
\mathbf{F}^{\prime t}
\end{array}\right\}\right)
$$

until a tolerance condition is obtained. This tolerance condition is

$$
\left|([\mathbf{M}]-[\mathbf{N}]) \mathbf{X}_{i}-\left\{\begin{array}{l}
\mathbf{F}^{\prime s} \\
\mathbf{F}^{\prime t}
\end{array}\right\}\right| \leqslant \varepsilon .
$$

The value of $\mathbf{X}_{i}$ when this tolerance is reached is the approached solution of the problem.

The first step of this technique is the modal calculation (now two modal basis have to be found) and the projection of the matrices to obtain $\left[\mathbf{C}_{2}\right]$. As noted previously these operations are performed on real frequency independent matrices of size $n$ and are executed only once. The second step is the resolution of the complex system of size $2 n$ (17) at each frequency. The numerical cost of this technique is linked to the number of iterations at each frequency. This number cannot be predicted a-priori but the closer from the solution of the problem the initial vector is, the smaller the number of iteration is. Hence for multiple frequency calculations, solution vector at the previous frequency can be used as the initial vector for the current frequency. In the case of frequencies close to each other, solution is not greatly modified and this method do not need a lot of iterations at each frequency and will allow a great reduction of calculation time. Not that the convergence of this method is linked to the norm of $[\mathbf{M}]^{-1}[\mathbf{N}]$ matrix. A test is performed in our programs. We do not have a proof to ensure that we can reach convergence for all cases but we want to mention that it was the case in practice on all cases we have tried.

\section{Semi-analytical method to purely com- pressional motions}

This section is concerned with the presentation of an analytical solver for numerical system (8) in configurations 
for which only purely compressional motions are considered. It is the case or example for 1D configurations. The validity of this assumption in $3 \mathrm{D}$ cases and its applications has been enlightened by Chazot an Guyader [17]. For this type of problems, the $N\left[\mathbf{K}_{1}\right]$ term can be neglected in (8). In this section linear finite-element are involved.

The method is first presented for a single porous structure problem. Secondly, the case of several structures is described. The coupling between substructures is taken into account by the way of Lagrange multipliers. Two monodimensional configurations are presented as an illustration: a single porous layer and a coupled porous-air system to enlighten the accounting of coupled substructures. Finite element results are compared with purely-analytical solution of the problem that can be found for such problem by the way of plane waves techniques.

\subsection{Single porous structure}

\subsubsection{Analytical resolution of the system for a single structure}

The solid and total displacement are solution of the linear system (8) where only $\left[\mathbf{M}_{0}\right]$ and $\left[\mathbf{K}_{0}\right]$ are needed. The eigenvector and eigenvalue matrices $\left[\boldsymbol{\Phi}_{0}\right]$ and $\left[\mathbf{k}_{0}^{2}\right]$ can then be obtained and the projection of (8) on the modal basis leads to the system (12) which in our case reads

$$
\begin{array}{r}
\left(\left[\begin{array}{cc}
\hat{P}\left[\mathbf{k}_{0}^{2}\right] & {[0]} \\
{[0]} & \widetilde{K}_{e q}\left[\mathbf{k}_{0}^{2}\right]
\end{array}\right]-\omega^{2}\left[\begin{array}{cc}
\tilde{\rho}_{s}[\mathbf{I}] & \tilde{\gamma} \tilde{\rho}_{e q}[\mathbf{I}] \\
\tilde{\gamma} \tilde{\rho}_{e q}[\mathbf{I}] & \tilde{\rho}_{e q}[\mathbf{I}]
\end{array}\right]\right) \\
\cdot\left\{\begin{array}{c}
\mathbf{q}^{s} \\
\mathbf{q}^{t}
\end{array}\right\}=\left\{\begin{array}{l}
\mathbf{F}^{\prime s} \\
\mathbf{F}^{\prime t}
\end{array}\right\} .
\end{array}
$$

It can be noticed that this system exhibits a decoupling of the unknowns $\left\{q_{i}^{s}, q_{i}^{t}\right\}$ which are solution of the two equations

$$
\begin{aligned}
\hat{P} k_{i}^{2} q_{i}^{s}-\omega^{2}\left(\widetilde{\rho}_{s} q_{i}^{s}+\tilde{\gamma} \tilde{\rho}_{e q} q_{i}^{t}\right) & =F_{i}^{\prime s}, \\
\widetilde{K}_{e q} k_{i}^{2} q_{i}^{t}-\omega^{2}\left(\tilde{\gamma} \widetilde{\rho}_{e q} q_{i}^{s}+\tilde{\rho}_{e q} q_{i}^{t}\right) & =F_{i}^{\prime t} .
\end{aligned}
$$

Hence $q_{i}^{s}$ and $q_{i}^{t}$ can be obtained analytically by solving these equations:

$$
q_{i}^{s}=p_{i, 1} F_{i}^{1}+p_{i, 2} F_{i}^{2}, \quad q_{i}^{t}=\mu_{1} p_{i, 1} F_{i}^{1}+\mu_{2} p_{i, 2} F_{i}^{2} .
$$

with

$$
p_{i, j}=\frac{1}{k_{i}^{2}-\delta_{j}^{2}}, \quad F_{i}^{j}=\frac{F_{i}^{\prime s}+\mu_{j} F_{i}^{\prime t}}{\hat{P}+\widetilde{K}_{e q} \mu_{j}^{2}} .
$$

$\delta_{i}$ and $\mu_{i}$ are wave numbers and ratio of the total displacement over the solid one of wave $i$ defined by Dazel et al. [11]. In these last expressions $p_{i, j}$ is the polar form associated to mode $i$ and relative to wave $j$. Even if the notion of resonance is not obvious as $\delta_{j}$ is a complex and frequency dependent function, these functions are linked to the mechanical resonances of the medium. The modal forces $F_{i}^{j}$ involves two contributions relative to both phases and the contribution of each phase is linked to the ration $\mu_{i}$ of the two displacements. The denominator of $F_{i}^{j}$ is a normalization factor linked to the stiffness. Thereby the solid and

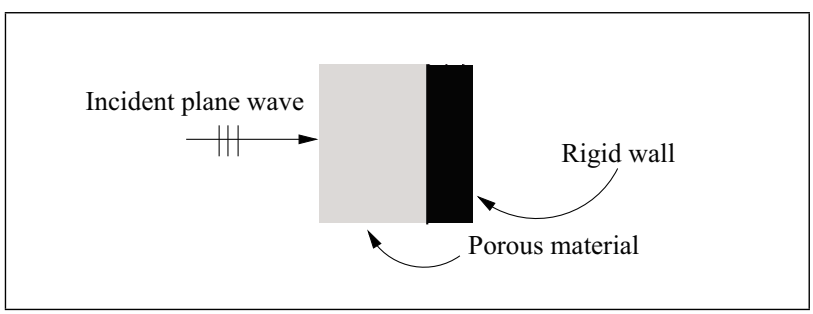

Figure 1. Porous material $A$ of infinite lateral dimensions (1D case) backed by an impervious rigid wall.

Table I. Parameters of porous material $A$ and $B$.

\begin{tabular}{|l|c|c|}
\hline \multicolumn{1}{|c|}{ Material: } & A & B \\
\hline Porosity $\phi$ & 0,97 & 0,97 \\
Flow resistivity $\sigma\left(\mathrm{N} \mathrm{m}^{-4} s\right)$ & 87000 & 40000 \\
Tortuosity $\alpha_{\infty}$ & 1,52 & 1,06 \\
Viscous charact. length $\Lambda(\mathrm{m})$ & $3.710^{-5}$ & $0,5610^{-4}$ \\
Thermal charact. length $\Lambda^{\prime}(\mathrm{m})$ & $1.210^{-4}$ & $0,11210^{-3}$ \\
Density $\rho_{1}\left(\mathrm{Kg} / \mathrm{m}^{3}\right)$ & 31 & 130 \\
Young modulus $E(\mathrm{~Pa})$ & $1,4310^{7}$ & $0,4410^{7}$ \\
Loss factor $\eta_{s}$ & 0,055 & 0, \\
Poisson coefficient $v$ & 0,3 & 0,1 \\
\hline
\end{tabular}

total contributions are expressed in (24a) as the sum of two similar parts, each one associated to the two Biot waves. Expressions (24a) analytically provides the modal amplitudes and the solid and total displacements are then obtained.

\subsubsection{Presentation of the problem}

The first configuration of interest is a single porous layer of material A of thickness $5 \mathrm{~cm}$ and infinite lateral dimensions backed by a rigid impervious wall (see Figure 1). An harmonic unit force is applied on the free face of the porous layer. Properties of the porous material are given in Table I. Absorption coefficient is plotted in Figure 2. An analytical solution is calculated through the analytical surface impedance which can be found in many references $[3,11]$. The numerical solution is a purely FEM with a direct solver in $\left\{\mathbf{u}^{s}, \mathbf{u}^{t}\right\}$ formulation. As the analytical and $\left\{\mathbf{u}^{s}, \mathbf{u}^{t}\right\}$ formulation results perfectly match, this example provides a validation of the formulation. Two main resonances can be observed (around $500 \mathrm{~Hz}$ and $1300 \mathrm{~Hz}$ ), the second one being more damped than the first one. In both cases, the resonance is associated with a particular phenomenon that can be called "decrease-increase". While increasing frequency there is first a decrease of the absorption coefficient followed by a brutal increase. It has been observed in several cases when Biot's effects are involved. In the case of motionless solid frame (equivalent fluid) the resonance just corresponds to a maximum of absorption. It is interesting to analyze this effect by the way of a modal approach.

The polar functions $p_{1}$ and $p_{2}$ associated to mode 1 and mode 2 are presented in Figure 3 (module) and 4 (phase). Note that the frequency limits are not the same in modulus and phase. For mode 1, a resonance of wave 2 is observed 


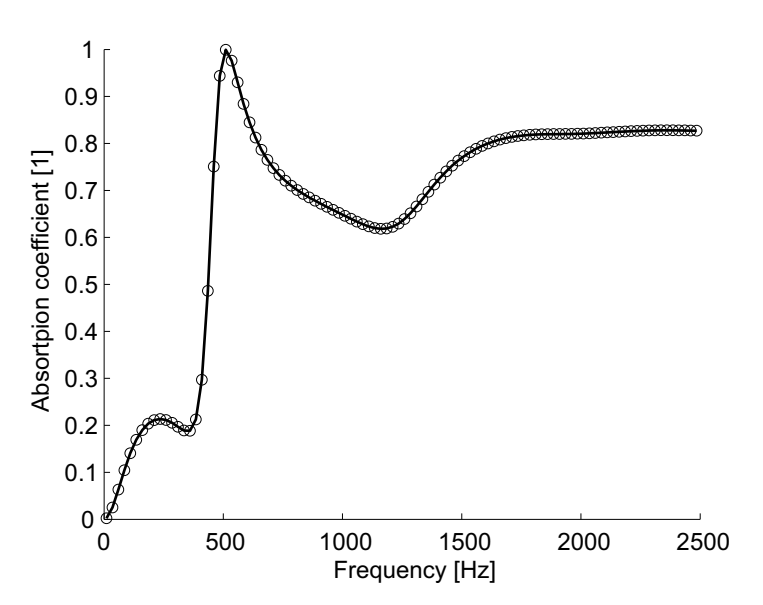

Figure 2. Absorption coefficient (continuous) analytical, $o$ finite element $\left\{\mathbf{u}^{s}, \mathbf{u}^{t}\right\}$ formulation.

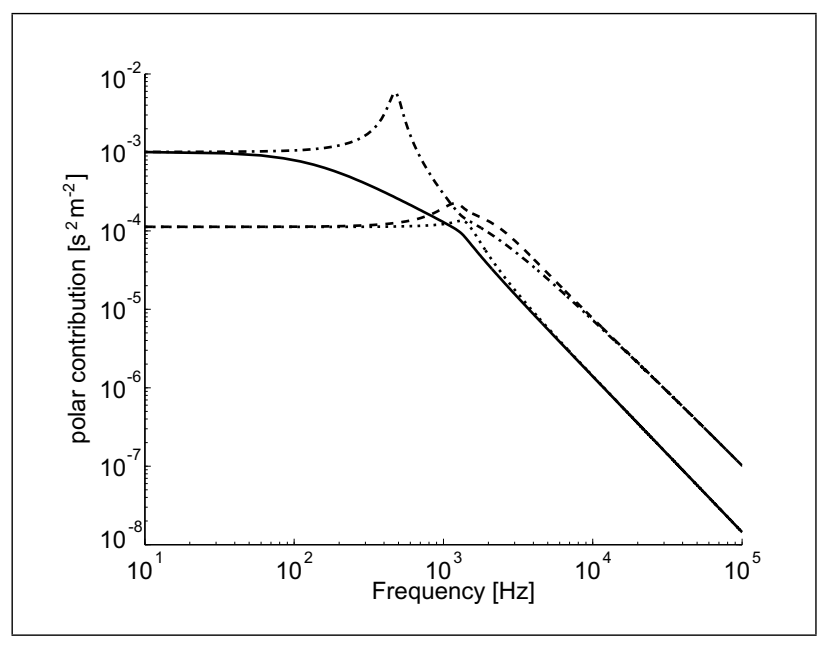

Figure 3. Amplitude of polar functions. solid: pole associated to wave 1 and mode 1; dash-dot: pole associated to wave 2 and mode 1;dot: pole associated to wave 1 and mode 2; dash: pole associated to wave 2 and mode 2 .

at $485 \mathrm{~Hz}$. At this frequency, one has $\delta_{1}=49.5-38.6 \mathrm{i}$ and $\delta_{2}=31.52-2.78 \mathrm{i}$ and the value of the first eigenvalue of the problem $k_{1}=31.4$. Wave 2 is mainly associated with the solid and is not as damped as the fluid one. That is the reason why no resonance is observed for pole 1 which is over damped. For the second mode, the resonance is at $1185 \mathrm{~Hz}$ and $\delta_{1}=90.9-40.6 \mathrm{i}$ and $\delta_{2}=75.3-18.0 \mathrm{i}$ with $k_{2}=94.2$. Hence, the two observed resonances at 500 and $1300 \mathrm{~Hz}$ are linked to the resonances of the modes. Nevertheless it does not explain the decrease-increase phenomenon. In order to better understand it is necessary to remind that the main dissipation mechanism in porous material is viscous effects when the relative motion of the fluid to the solid is important; hence there won't be viscous effects if the solid and fluid displacement are equal even if they are important. The solid and total contribution of mode 1 and 2 are shown in Figure 5 (module) and 6 (phase). One can see that at the resonance of mode 1 the complex contribution of the solid and total are quite equal

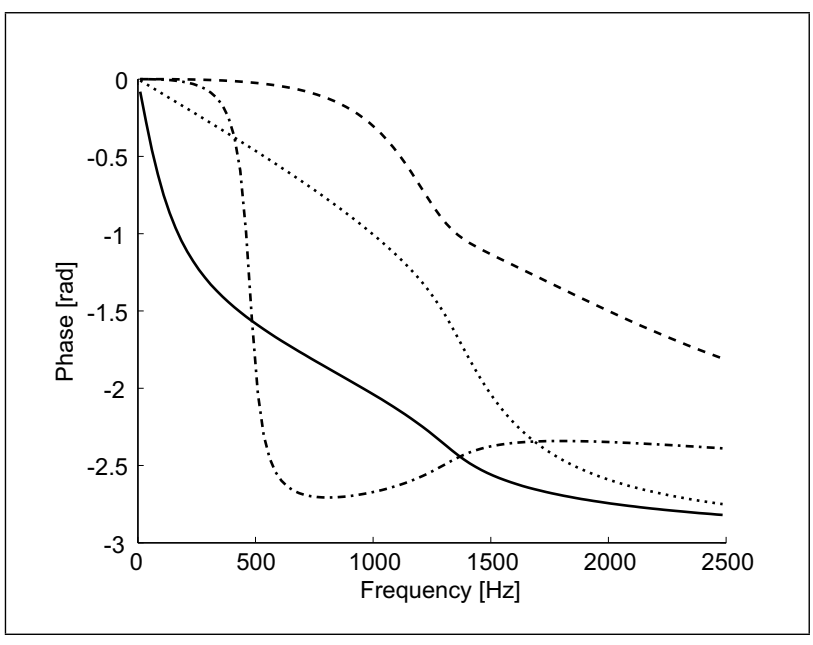

Figure 4. Phase of polar functions. solid: pole associated to wave 1 and mode 1; dash-dot: pole associated to wave 2 and mode 1 ; dot: pole associated to wave 1 and mode 2; dash: pole associated to wave 2 and mode 2 .

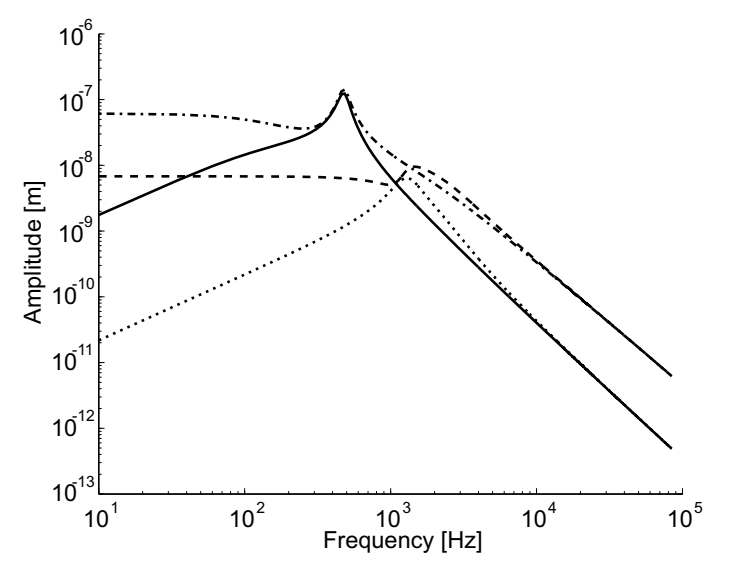

Figure 5. Amplitude of modal contributions. solid: solid contribution of mode 1; dash-dot: total contribution of mode 1; dot: solid contribution of mode 2; dash: total contribution of mode 2 .

(in module and phase). Consequently at this frequency the solid and total displacement are close (5\% difference in modulus) as $u^{t}=(1-\phi) u^{s}+\phi u^{f}$, the solid and fluid displacements are close thereby reducing viscous effects. The important variation of phase few $\mathrm{Hz}$ after the resonance induces an important amplitude variation and a different phase, there is then a significant relative motion and this corresponds to the maximum of absorption.

\subsection{Multilayered porous structure}

\subsubsection{Presentation of the problem}

A multilayered problem is now considered. The studied configuration is a porous material $A$ of thickness $10 \mathrm{~cm}$ and an air gap of the same thickness backed by an impervious rigid wall (see Figure 7). The lateral dimension 


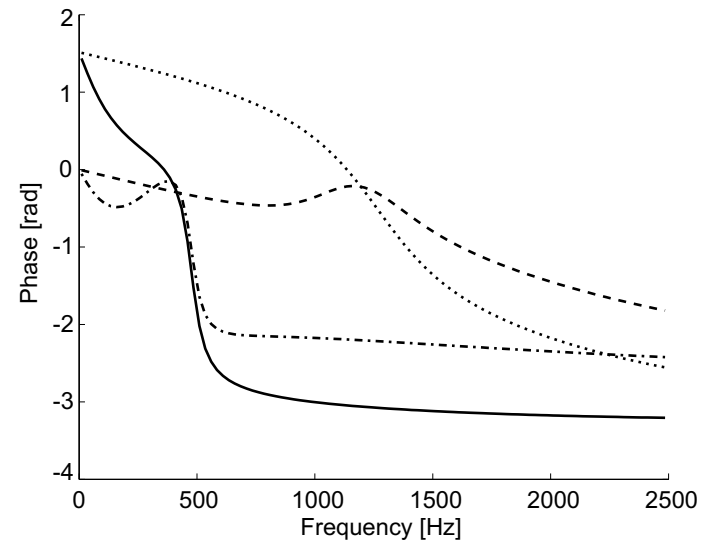

Figure 6. Phase of modal contributions. solid: solid contribution of mode 1; dash-dot: total contribution of mode 1; dot: solid contribution of mode 2; dash: total contribution of mode 2 .

of the structure is considered infinite (1D case). An harmonic plane wave of unity pressure amplitude impinges the porous layer with normal incidence.

\subsubsection{Finite element modeling and resolution}

The air and porous structure are both modelled with linear finite element. Let $\mathbf{u}^{s}, \mathbf{u}^{t}$ and $\mathbf{u}^{a}$ be respectively the discretized solid and total displacements of the porous and the discretized displacement of air. The displacement continuity relation is imposed through Lagrange multipliers: the common displacement at the boundary corresponds to two degrees of freedom, one for each substructure. The equality is ensured by an additional condition. It leads to the following linear system:

$$
\begin{aligned}
& \left(\left[\begin{array}{cc|c|c}
\hat{P}\left[\mathbf{K}_{0}\right] & {[\mathbf{0}]} & {[\mathbf{0}]} & \lambda_{s} \\
{[\mathbf{0}]} & \widetilde{K}_{e q}\left[\mathbf{K}_{0}\right] & {[\mathbf{0}]} & \lambda_{t} \\
\hline[\mathbf{0}] & {[\mathbf{0}]} & K_{0}\left[\mathbf{K}_{a}\right] & \lambda_{a} \\
\hline{ }^{t} \lambda_{s} & { }^{t} \lambda_{t} & { }^{t} \lambda_{a} & \lambda_{c}
\end{array}\right]\right. \\
& \left.-\omega^{2}\left(\begin{array}{cc|c|c}
\tilde{\rho}_{s}\left[\mathbf{M}_{0}\right] & \tilde{\gamma} \tilde{\rho}_{e q}\left[\mathbf{M}_{0}\right] & {[\mathbf{0}]} & \mathbf{0}_{s} \\
\tilde{\gamma} \tilde{\rho}_{e q}\left[\mathbf{M}_{0}\right] & \tilde{\rho}_{e q}\left[\mathbf{M}_{0}\right][\mathbf{0}] & {[\mathbf{0}]} & \mathbf{0}_{t} \\
\hline[\mathbf{0}] & {[\mathbf{0}]} & \rho_{0}\left[\mathbf{M}_{a}\right] & \mathbf{0}_{a} \\
\hline{ }^{t} \mathbf{0}_{s} & { }^{t} \mathbf{0}_{t} & { }^{t} \mathbf{0}_{a} & { }^{t} \mathbf{0}_{c}
\end{array}\right]\right) \\
& \left\{\begin{array}{l}
\mathbf{u}^{s} \\
\mathbf{u}^{t} \\
\mathbf{u}^{a} \\
F^{c}
\end{array}\right\}=\left\{\begin{array}{l}
\mathbf{F}^{s} \\
\mathbf{F}^{t} \\
\mathbf{F}^{a} \\
u^{c}
\end{array}\right\}
\end{aligned}
$$

$\left[\mathbf{K}_{a}\right]$ and $\left[\mathbf{M}_{a}\right]$ are respectively the stiffness and mass matrices of the air. $K_{0}$ and $\rho_{0}$ correspond to the compressibility and density of air. $\lambda_{t}$ is a vector in which the only nonnull component is a 1 at the index corresponding to the degree of freedom of the boundary. $\lambda_{a}$ is a vector in which the only non-null component is a -1 at the index corresponding to the degree of freedom of the boundary. $F_{c}$ is an additional unknown of the problem. It can be shown that it corresponds to the interaction force between the porous and air structure. $\lambda_{s}, u_{c}$ and $\lambda_{c}$ are null. The last line equation simply gives the continuity relation.

This system can also be solved analytically by the way of normal modes. The first step is the projection of system (25) on the following modal basis defined by

$$
[\boldsymbol{\Phi}]=\left[\begin{array}{lllll}
{\left[\boldsymbol{\Phi}_{0}\right]} & & & \\
& {\left[\boldsymbol{\Phi}_{0}\right]} & & \\
& & {\left[\boldsymbol{\Phi}_{a}\right]} & \\
& & & 1
\end{array}\right],
$$

where $\left[\boldsymbol{\Phi}_{0}\right]$ (resp. $\left.\left[\boldsymbol{\Phi}_{a}\right]\right)$ are the normal modes associated with $\left[\mathbf{M}_{0}\right]$ and $\left[\mathbf{K}_{0}\right]$ (resp. $\left[\mathbf{M}_{a}\right]$ and $\left[\mathbf{K}_{a}\right]$ ) and normalize with respect to $\left[\mathbf{M}_{0}\right]$ (resp. $\left[\mathbf{M}_{a}\right]$ ). These modes are associated with the eigenvalues $\mathbf{k}_{0}^{2}$ (resp. $\mathbf{k}_{a}^{2}$ ). The following system is then obtained,

$$
\left[\begin{array}{cc|c|c}
{\left[\boldsymbol{\Delta}_{s}\right]} & {\left[\boldsymbol{\Delta}_{c}\right]} & {[\mathbf{0}]} & \lambda_{s}^{\prime} \\
{\left[\boldsymbol{\Delta}_{c}\right]} & {\left[\boldsymbol{\Delta}_{t}\right]} & {[\mathbf{0}]} & \lambda_{t}^{\prime} \\
\hline[\mathbf{0}] & {[\mathbf{0}]} & {\left[\boldsymbol{\Delta}_{a}\right]} & \lambda_{a}^{\prime} \\
\hline{ }^{t} \lambda_{s}^{\prime} & { }^{t} \lambda_{t}^{\prime} & { }^{t} \lambda_{a}^{\prime} & \lambda_{c}
\end{array}\right]\left\{\begin{array}{l}
\mathbf{q}^{s} \\
\mathbf{q}^{t} \\
\mathbf{q}^{a} \\
F^{c}
\end{array}\right\}=\left\{\begin{array}{c}
\mathbf{F}_{p}^{s} \\
\mathbf{F}_{p}^{t} \\
\mathbf{F}_{p}^{a} \\
u^{c}
\end{array}\right\} .
$$

The $\left[\boldsymbol{\Delta}_{i}\right]$ diagonal matrices are associated with the following vectors,

$$
\begin{aligned}
\boldsymbol{\Delta}_{s} & =\hat{P} \mathbf{k}_{0}^{2}-\omega^{2} \widetilde{\rho}_{s}, \quad \boldsymbol{\Delta}_{t}=\widetilde{K}_{e q} \mathbf{k}_{0}^{2}-\omega^{2} \widetilde{\rho}_{e q}, \\
\boldsymbol{\Delta}_{a} & =K_{0} \mathbf{k}_{a}^{2}-\omega^{2} \rho_{0}, \quad \boldsymbol{\Delta}_{c}=-\omega^{2} \widetilde{\gamma} \widetilde{\rho}_{e q} .
\end{aligned}
$$

$\lambda^{\prime}$ and $\mathbf{F}_{p}$ vectors are the projections of $\lambda$ and $\mathbf{F}$ vectors on the modal basis. System (27) is mainly built from diagonal matrices corresponding to the decoupling of normal modes, nevertheless a coupling is introduced by the continuity relations (denoted here by the Lagrange multipliers $\lambda^{\prime}$ ). In order to take benefit of the diagonal structures, the second step of the resolution method is to first seek for the value of $F_{c}$. The idea is to replace the last line of system (27) by an adequate linear combination of the first lines of the matrix. It is a procedure inspired from Gauss elimination classical method to solve a linear system. It is detailed in appendix $\mathrm{A} 3$ and one obtains

$$
F_{c}=\frac{u_{c}-\sum \frac{L_{i} F_{i}}{\Delta_{i}}}{\lambda_{c}-\sum \frac{L_{i} L_{i}^{\prime}}{\Delta_{i}}},
$$

where $\mathbf{L}, \mathbf{L}, \mathbf{F}$ and $\boldsymbol{\Delta}$ are defined in appendix A3. As $F_{c}$ is known, the last column of matrix (27) can be sent to the second member of the equation and one has

$$
\left[\begin{array}{cc|c}
{\left[\boldsymbol{\Delta}_{s}\right]\left[\boldsymbol{\Delta}_{c}\right]} & {[\mathbf{0}]} \\
{\left[\boldsymbol{\Delta}_{c}\right]\left[\boldsymbol{\Delta}_{t}\right]} & {[\mathbf{0}]} \\
\hline[\mathbf{0}] & {[\mathbf{0}]} & {\left[\boldsymbol{\Delta}_{a}\right]}
\end{array}\right]\left\{\begin{array}{c}
\mathbf{q}^{s} \\
\mathbf{q}^{t} \\
\mathbf{q}^{a}
\end{array}\right\}=\left\{\begin{array}{c}
\mathbf{F}_{p}^{s}-\lambda_{s}^{\prime} F_{c} \\
\mathbf{F}_{p}^{t}-\lambda_{t}^{\prime} F_{c} \\
\mathbf{F}_{p}^{a}-\lambda_{a}^{\prime} F_{c}
\end{array}\right\} .
$$

This system can be solved analytically with the method of subsection 4.1.1 for the porous structure. For the air layer, a classical modal resolution is performed.

Figure 8 presents the displacements of both porous and air layer at $1500 \mathrm{~Hz}$. The finite element resolution involving 50 linear elements for both media is in perfect agreement with analytical results. The continuity of air/total displacement of the porous material can be illustrate in this 


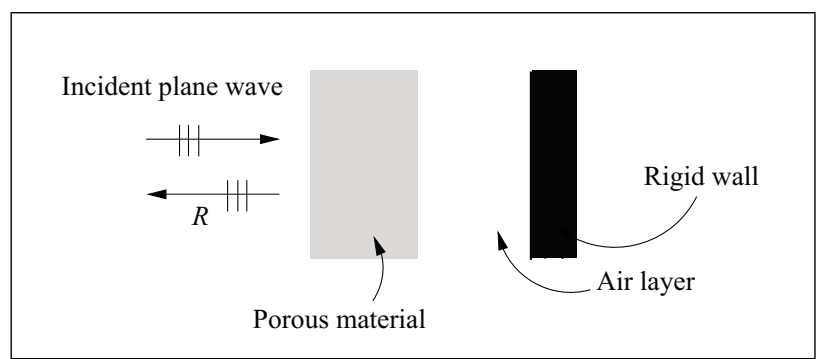

Figure 7. A layered material comprising a porous material $A$ of infinite lateral dimensions (1D case) and a layer of air backed by an impervious rigid wall.



Figure 8. Analytical and finite element resolution of the multilayer problem. Solid: analytical solution; o: solid displacement of the porous; $\nabla$ : total displacement of the porous; + + displacement of air.

example. The numerical solution is both computed with the proposed analytical modal resolution and a direct resolution of system (25) which exactly coincide. These limits are observed also in the case of a similar resolution with $\left\{\mathbf{u}^{s}, \mathbf{u}^{f}\right\}$ formulation and even more for $\left\{\mathbf{u}^{s}, P\right\}$ formulation. The reason of a better conditioning of the two steps in the proposed approach (obtainment of the Lagrange multiplier and analytical resolution of the system) is that each type (physical nature) of dof is solved separately from the others. Similar resonance behavior than the one observed on a single porous structure are observed for this problem but are not presented here. The proposed resolution method involves all the modes. It is sometimes useful to consider the truncation on a modal sub-family. As a title of example, Figure 9 presented the module truncated solution by using the first three modes as well as their individual contribution. It can be seen that this approximation is satisfactory except around the porous-air interface where the contribution of higher modes is needed. As the analytical resolution to obtain the modal contributions is not really consuming, there is in this case no reason to perform a truncation, but it is a perspective for future works.



Figure 9. Decomposition of the numerical solution by truncation after the first three modes. Solid: analytical solution; dot: first mode; dash: second mode; dash-dot: third mode; 'o': modal solution.

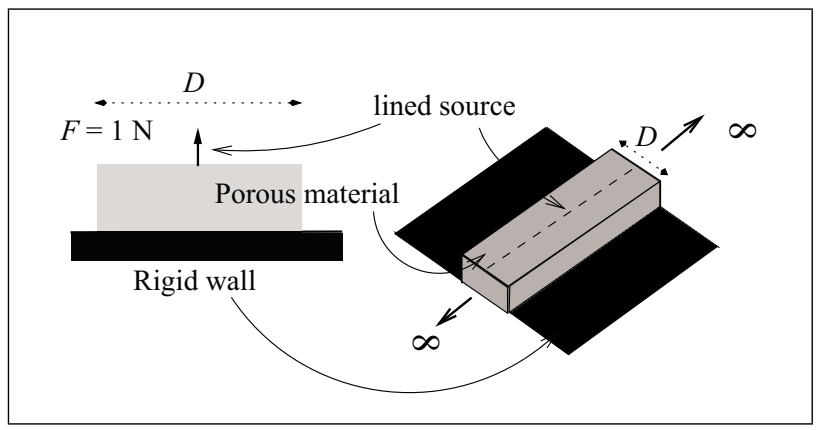

Figure 10. A layer of porous material (porous $B$ ) of semi-infinite lateral dimension (2D case) of width $D$ backed by an impervious rigid wall.

\section{Two dimensional problem}

In the preceding section, only $1 \mathrm{D}$ problems were considered. The aim of this part is to compare the efficiency of the proposed numerical methods of section 3 to classical ones. A 2D problem is now considered.

\subsection{Presentation of the problem}

The studied configuration is depicted in Figure 10. A block of porous material of material $B$ of section $10 \mathrm{~cm}$ by $10 \mathrm{~cm}$ and infinite lateral extent is bonded onto a impervious rigid wall. It is excited by a line force of $1 \mathrm{~N} / \mathrm{m}$ in the middle of his top face. As this problem is infinite in one direction, it can be modelled through a 2D approximation. Triangular isoparametric quadratic elements are used for the discretization and a Delaunay mesh triangulation is first performed for several mesh refinements. The more refined is the mesh and of better quality the result is, higher the numerical cost is. 


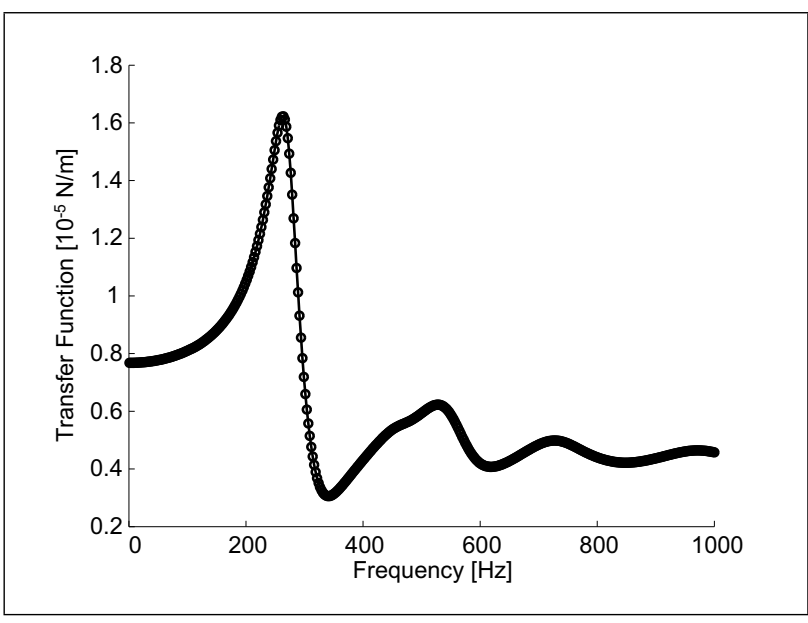

Figure 11. Transfer function of the displacement over the force at the node of excitation. solid: $\left\{\mathbf{u}^{s}, \mathbf{u}^{f}\right\}$ formulation; o $\left\{\mathbf{u}^{s}, \mathbf{u}^{t}\right\}$ formulation; * $\left\{\mathbf{u}^{s}, P\right\}$ formulation.

\subsection{Results}

\subsubsection{Validation of the formulation}

In order to present another validation of the $\left\{\mathbf{u}^{s}, \mathbf{u}^{t}\right\}$ formulation, Figure 11 presents the transfer function of the excitation node for different formulations. This transfer function is defined as the total displacement of the porous material at the node over the force for different frequencies. The considered formulations are $\left\{\mathbf{u}^{s}, \mathbf{u}^{f}\right\},\left\{\mathbf{u}^{s}, \mathbf{u}^{t}\right\}$ and the classical $\left\{\mathbf{u}^{s}, P\right\}$ ones. The mesh is composed of 1680 triangular quadratic elements and the approximate size of the elements is $2.6 \mathrm{~mm}$ and corresponding approximatively to a $\lambda / 15$ discretization of the shear wave which is the most discriminative one. It is mentioned that this mesh is only considered as a convergence example. A perfect agreement is shown between these 3 formulations thereby showing an additional validation of the proposed formulation compared to $\left\{\mathbf{u}^{s}, \mathbf{u}^{f}\right\}$ and $\left\{\mathbf{u}^{s}, P\right\}$ ones.

A comparison of computational time is presented in Figure 12. The transfer function is computed for 400 frequencies linearly spaced between 1 and $1000 \mathrm{~Hz}$ by the way of $\left\{\mathbf{u}^{s}, \mathbf{u}^{f}\right\},\left\{\mathbf{u}^{s}, P\right\}$ as well as $\left\{\mathbf{u}^{s}, \mathbf{u}^{t}\right\}$ formulation with a direct and iterative resolution method. Programs are written in Fortran and sparse algorithms are used to calculate eigenpairs and the direct solutions of the systems.

As the computational time mainly depends on the calculator and its configuration, computation times won't be directly presented. It is preferred to compare each one of them to the time of the $\left\{\mathbf{u}^{s}, \mathbf{u}^{f}\right\}$ formulation chosen as a reference. Hence, for each number of elements, the computational time of the $\left\{\mathbf{u}^{s}, \mathbf{u}^{f}\right\}$ formulation is divided by the one of the other method. The results are presented in Figure 12. For example, for 2200 elements, the computation with the Porous-Jacobi method is 5.5 times faster than the $\left\{\mathbf{u}^{s}, \mathbf{u}^{f}\right\}$ method.

For each method, the time are calculated through the cpu_time() function. Matrices are first allocated and matrices $\left[\mathbf{M}_{0}\right],\left[\mathbf{K}_{0}\right]$ and $\left[\mathbf{K}_{1}\right]$ are calculated. This first step is of course common to all the solver procedures. Hence,

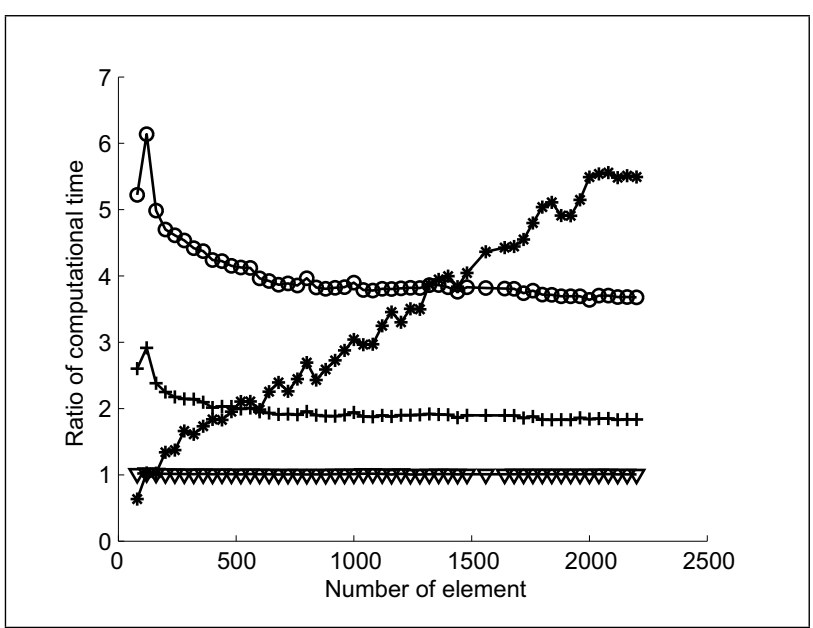

Figure 12. ( $\nabla)\left\{\mathbf{u}^{s}, \mathbf{u}^{f}\right\}$ formulation with direct solver; (+) $\left\{\mathbf{u}^{s}, P\right\}$ formulation with direct solver; $(o)\left\{\mathbf{u}^{s}, \mathbf{u}^{t}\right\}$ formulation with the proposed direct modal resolution; $(*)\left\{\mathbf{u}^{s}, \mathbf{u}^{t}\right\}$ formulation with the proposed iterative modal resolution.

Table II. Computational time for the example of Figure 11 for the different methods.

\begin{tabular}{|c|c|}
\hline Method & Time in seconds \\
\hline$\left\{\mathbf{u}^{s}, \mathbf{u}^{f}\right\}$ & 3595 \\
$\left\{\mathbf{u}^{s}, P\right\}$ & 1896 \\
$\left\{\mathbf{u}^{s}, \mathbf{u}^{t}\right\}$ (direct) & 3563 \\
$\left\{\mathbf{u}^{s}, \mathbf{u}^{t}\right\}$ (modal direct) & 944 \\
$\left\{\mathbf{u}^{s}, \mathbf{u}^{t}\right\}$ (modal iterative) & 809 \\
\hline
\end{tabular}

the considered computational times only considers the resolution methods. For modal method the initial mode computation(s) is of course taken into account. As an illustration, the computational times for the result of Figure 11 are given in Table II.

It can be seen that $\left\{\mathbf{u}^{s}, \mathbf{u}^{f}\right\}$ and $\left\{\mathbf{u}^{s}, \mathbf{u}^{t}\right\}$ with a direct solver methods are equivalent in terms of computational time. Even if it is not significative, $\left\{\mathbf{u}^{s}, \mathbf{u}^{t}\right\}$ is a bit faster (a few seconds for the example of Table II). Those seconds corresponds to the time of the assembling of stiffness coupling for the $\left\{\mathbf{u}^{s}, \mathbf{u}^{f}\right\}$ formulation. $\left\{\mathbf{u}^{s}, \boldsymbol{P}\right\}$ formulation is around 2 times quicker than the $\left\{\mathbf{u}^{s}, \mathbf{u}^{f}\right\}$ method due to the reduction of dof per node. The proposed direct modal resolution exhibits a calculation 4 times faster and the porousJacobi methods is shown to be more and more efficient as the number of elements increases. The authors want to enlighten that this efficiency studies are a first step and that further studies must be done in particular to find the order of computations used in the different approaches

\section{Conclusion}

An alternative displacement finite element formulation of the Biot theory for poroelastic materials and three resolution methods have been presented. The poroelastic formulation is based on the solid and total displacement degrees of freedom. This formulation has the advantage of a diago- 
nal by block stiffness matrix together with natural boundary conditions while the porous medium is coupled with air or an other porous medium. The methods have been validated in comparison with analytical model on two 1D configurations involving a porous layer bonded onto a hard backing, and with an air gap to demonstrate the ability to handle coupled systems. For porous medium for which the shear can be neglected, an analytical solver allows the definition of normal modes for porous materials. The efficiency of our method has also been demonstrated in terms of computation time: the direct solver with the proposed formulation is 2 times quicker than with the mixed displacement-pressure formulation. The iterative method is even more efficient when the size of the system increases allowing to manage large size applications. A follow-up of this study is to investigate modal truncation or to apply mass-condensation techniques.

\section{Appendix}

\section{A1. Topics on Biot-Allard model}

This appendix provides the expressions of the inertial and constitutive parameters of the Biot-Allard model. All these expressions can be found in Allard [3]. This model allows to find the expressions of the coefficient used in the manuscript as a function of the material properties of Table I. These expressions are given for a circular frequency $\omega$.

The density terms are first reminded. They are given by

$$
\begin{aligned}
\rho_{1} & =(1-\phi) \rho_{s}, \quad \rho_{2}=\phi \rho_{0}, \\
\rho_{12} & =-\phi \rho_{0}\left(\alpha_{\infty}-1\right) .
\end{aligned}
$$

$\phi$ is porosity, $\rho_{s}$ is skeleton material density, $\rho_{0}$ is interstitial fluid density and $\alpha_{\infty}$ refers to geometric tortuosity. $\rho_{12}$ accounts for the interaction between the inertia forces of the solid and fluid phase. The apparent inertial mass can be introduced,

$$
\tilde{\rho}_{12}=\rho_{12}-\frac{\tilde{b}}{j \omega}, \quad \tilde{\rho}_{22}=\rho_{2}-\tilde{\rho}_{12} .
$$

The viscous effects are modelled through $\tilde{b}$ coefficient whose expression is

$$
\widetilde{b}=\mathrm{j} \omega \phi \rho_{0}\left(\widetilde{\alpha}-\alpha_{\infty}\right),
$$

$\widetilde{\alpha}$ is the dynamic tortuosity defined by

$$
\tilde{\alpha}=1-\frac{j \phi \sigma}{\alpha_{\infty} \rho_{0} \omega} \sqrt{1-\frac{4 j \alpha_{\infty}^{2} \eta_{a} \rho_{0} \omega}{(\sigma \Lambda \phi)^{2}}} .
$$

$\sigma$ is the flow resistivity, $\eta_{a}$ is the dynamic viscosity of air and $\Lambda$ is the viscous characteristic length. The equivalent density $\tilde{\rho}_{e q}$ and coupling coefficient are given by

$$
\tilde{\rho}_{e q}=\frac{\tilde{\rho}_{22}}{\phi^{2}}, \quad \tilde{\gamma}=\phi\left(\frac{\widetilde{\rho}_{12}}{\tilde{\rho}_{22}}-\frac{1-\phi}{\phi}\right) .
$$

The thermal properties are given by the dynamic compressibility $\widetilde{K}_{e q}$,

$$
\widetilde{K}_{e q}=\frac{\gamma P_{0}}{\gamma-(\gamma-1)\left[1+\frac{8 \eta_{a}}{j \Lambda^{\prime} \operatorname{Pr} \omega \rho_{0}} \sqrt{1+\frac{j \rho_{0} \omega \operatorname{Pr} \Lambda^{\prime 2}}{16 \eta_{a}}}\right]}
$$

where $\Lambda^{\prime}$ is the thermal characteristic length, $\operatorname{Pr}$ is the Prandtl number, $P_{0}$ is the ambiant pressure, $\gamma$ is the ratio of specific heats of air. For sound absorbing materials, one has

$$
\widetilde{Q}=\phi(1-\phi) \widetilde{K}_{e q}, \quad \widetilde{R}=\phi^{2} \widetilde{K}_{e q}
$$

The structural mechanical parameters $N$ and $\hat{A}$ are given by

$$
N=\frac{E\left(1+j \eta_{s}\right)}{2(1+v)}, \quad \hat{A}=\frac{2 N v}{1-2 v}, \quad \hat{P}=\hat{A}+2 N
$$

The two compressional waves of the porous medium are defined by their wave number $\delta_{i}$ and the ratio of the total displacement over the solid one $\mu_{i}$. They are defined by

$$
\delta_{i}^{2}=\frac{\left(\delta_{s 2}^{2}+\delta_{e q}^{2}\right) \pm \sqrt{\left(\delta_{s 2}^{2}+\delta_{e q}^{2}\right)^{2}-4 \delta_{e q}^{2} \delta_{s 1}^{2}}}{2}
$$

with

$$
\begin{aligned}
\delta_{e q} & =\omega \sqrt{\frac{\tilde{\rho}_{e q}}{\widetilde{K}_{e q}}}, \quad \delta_{s 1}=\omega \sqrt{\frac{\tilde{\rho}}{\hat{P}}}, \\
\delta_{s 2} & =\omega \sqrt{\frac{\tilde{\rho}_{s}}{\hat{P}}}, \quad \tilde{\rho}=\rho_{1}-\tilde{\rho}_{12}-\frac{\widetilde{\rho}_{12}^{2}}{\rho_{2}-\widetilde{\rho}_{12}}, \\
\tilde{\rho} & =\tilde{\rho}_{s}-\widetilde{\gamma}^{2} \tilde{\rho}_{e q},
\end{aligned}
$$

and

$$
\mu_{i}=\tilde{\gamma} \frac{\left(\delta_{i}^{2}-\delta_{s 2}^{2}\right)}{\delta_{s 2}^{2}-\delta_{s 1}^{2}}=\tilde{\gamma} \frac{\delta_{e q}^{2}}{\delta_{i}^{2}-\delta_{e q}^{2}}
$$

\section{A2. Finite element}

\section{A2.1. Elementary matrices}

$$
\left[B_{s}\right]=\left[\begin{array}{cccccc}
\frac{\partial}{\partial x} & 0 & 0 & \frac{\partial}{\partial y} & 0 & \frac{\partial}{\partial z} \\
0 & \frac{\partial}{\partial y} & 0 & \frac{\partial}{\partial x} & \frac{\partial}{\partial z} & 0 \\
0 & 0 & \frac{\partial}{\partial z} & 0 & \frac{\partial}{\partial y} & \frac{\partial}{\partial x}
\end{array}\right]\left[N_{u}\right],
$$




$$
\begin{aligned}
& {\left[D_{0}\right]=\left[\begin{array}{llllll}
1 & 1 & 1 & 0 & 0 & 0 \\
1 & 1 & 1 & 0 & 0 & 0 \\
1 & 1 & 1 & 0 & 0 & 0 \\
0 & 0 & 0 & 0 & 0 & 0 \\
0 & 0 & 0 & 0 & 0 & 0 \\
0 & 0 & 0 & 0 & 0 & 0
\end{array}\right],} \\
& {\left[D_{1}\right]=\left[\begin{array}{cccccc}
0 & -2 & -2 & 0 & 0 & 0 \\
-2 & 0 & -2 & 0 & 0 & 0 \\
-2 & -2 & 0 & 0 & 0 & 0 \\
0 & 0 & 0 & 1 & 0 & 0 \\
0 & 0 & 0 & 0 & 1 & 0 \\
0 & 0 & 0 & 0 & 0 & 1
\end{array}\right]}
\end{aligned}
$$

\section{A2.2. Matrices for monodimensional problems}

$$
\begin{aligned}
& {\left[\mathbf{K}_{0}\right]=\frac{1}{h}\left[\begin{array}{ccccc}
1 & -1 & 0 & 0 & 0 \\
-1 & 2 & -1 & 0 & 0 \\
0 & \ddots & \ddots & \ddots & 0 \\
0 & \ddots & -1 & 2 & -1 \\
0 & 0 & 0 & -1 & 2
\end{array}\right],} \\
& {\left[\mathbf{M}_{0}\right]=\frac{h}{6}\left[\begin{array}{lllll}
2 & 1 & 0 & 0 & 0 \\
1 & 4 & 1 & 0 & 0 \\
0 & \ddots & \ddots & \ddots & 0 \\
0 & \ddots & 1 & 4 & 1 \\
0 & 0 & 0 & 1 & 4
\end{array}\right]}
\end{aligned}
$$

$h$ corresponds to the size of the elements.

\section{A3. Elimination of the Lagrange multiplier}

The aim of this section is to obtain the analytical value of the Lagrange multiplier $F_{c}(29)$ from linear system (27) which is now reminded,

$$
\left[\begin{array}{cc|c|c}
{\left[\boldsymbol{\Delta}_{s}\right]} & {\left[\boldsymbol{\Delta}_{c}\right]} & {[\mathbf{0}]} & \lambda_{s}^{\prime} \\
{\left[\boldsymbol{\Delta}_{c}\right]} & {\left[\boldsymbol{\Delta}_{t}\right]} & {[\mathbf{0}]} & \lambda_{t}^{\prime} \\
\hline[\mathbf{0}] & {[\mathbf{0}]} & {\left[\boldsymbol{\Delta}_{a}\right]} & \lambda_{a}^{\prime} \\
\hline{ }^{t} \lambda_{s}^{\prime} & { }^{t} \lambda_{t}^{\prime} & { }^{t} \lambda_{a}^{\prime} & \lambda_{c}
\end{array}\right]\left\{\begin{array}{c}
\mathbf{q}^{s} \\
\mathbf{q}^{t} \\
\mathbf{q}^{a} \\
F^{c}
\end{array}\right\}=\left\{\begin{array}{c}
\mathbf{F}_{p}^{s} \\
\mathbf{F}_{p}^{t} \\
\mathbf{F}_{p}^{a} \\
u^{c}
\end{array}\right\}
$$

The first step is to perform an elimination of the non diagonal block-matrices of the porous matrix. The previous system is then equivalent to

$$
\begin{gathered}
{\left[\begin{array}{cc|c|c}
{\left[\boldsymbol{\Delta}_{s}\right]} & {\left[\boldsymbol{\Delta}_{c}\right]} & {[\mathbf{0}]} & \lambda_{s}^{\prime} \\
{[\mathbf{0}]} & {\left[\boldsymbol{\Delta}_{t}\right]-\frac{\left[\boldsymbol{\Delta}_{c}\right]^{2}}{\left[\boldsymbol{\Delta}_{s}\right]}} & {[\mathbf{0}]} & \lambda_{t}^{\prime}-\frac{\left[\boldsymbol{\Delta}_{c}\right] \lambda_{s}^{\prime}}{\left[\boldsymbol{\Delta}_{s}\right]} \\
\hline[\mathbf{0}] & {[\mathbf{0}]} & {\left[\boldsymbol{\Delta}_{a}\right]} & \lambda_{a}^{\prime} \\
\hline{ }^{t} \lambda_{s}^{\prime} & { }^{t} \lambda_{t}^{\prime} & { }^{t} \lambda_{a}^{\prime} & \lambda_{c}
\end{array}\right]\left\{\begin{array}{c}
\mathbf{q}^{s} \\
\mathbf{q}^{t} \\
\mathbf{q}^{a} \\
F^{c}
\end{array}\right\}} \\
\\
=\left\{\begin{array}{c}
\mathbf{F}_{p}^{s} \\
\mathbf{F}_{p}^{t}-\frac{\left[\boldsymbol{\Delta}_{c}\right] \mathbf{F}_{p}^{s}}{\left[\boldsymbol{\Delta}_{s}\right]} \\
\mathbf{F}_{p}^{a} \\
u^{c}
\end{array}\right\} .
\end{gathered}
$$

In this equation, as the $[\boldsymbol{\Delta}]$ are diagonal matrices, the products and division of these matrices must be understood as term to term operations.

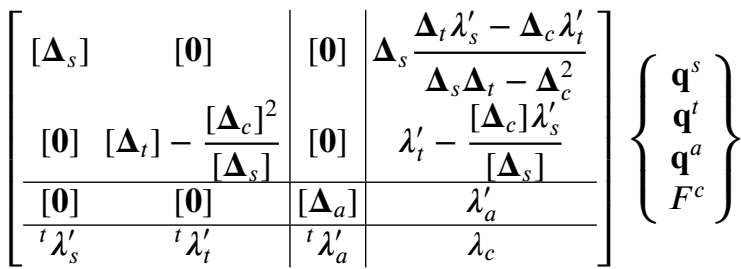

$$
\begin{aligned}
& =\left\{\begin{array}{c}
\boldsymbol{\Delta}_{s} \frac{\boldsymbol{\Delta}_{t} \mathbf{F}_{p}^{s}-\boldsymbol{\Delta}_{c} \mathbf{F}_{p}^{t}}{\boldsymbol{\Delta}_{s} \boldsymbol{\Delta}_{t}-\boldsymbol{\Delta}_{c}^{2}} \\
\mathbf{F}_{p}^{t}-\frac{\left[\boldsymbol{\Delta}_{c}\right] \mathbf{F}_{p}^{s^{2}}}{\left[\boldsymbol{\Delta}_{s}\right]} \\
\mathbf{F}_{p}^{a} \\
u^{c}
\end{array}\right\} .
\end{aligned}
$$

The following vectors are introduced:

$$
\begin{gathered}
\mathbf{L}=\left\{\begin{array}{c}
\lambda_{s}^{\prime} \\
\lambda_{t}^{\prime} \\
\lambda_{a}^{\prime}
\end{array}\right\}, \quad \mathbf{L}^{\prime}=\left\{\begin{array}{c}
\boldsymbol{\Delta}_{s} \frac{\boldsymbol{\Delta}_{t} \lambda_{s}^{\prime}-\boldsymbol{\Delta}_{c} \lambda_{t}^{\prime}}{\boldsymbol{\Delta}_{s} \boldsymbol{\Delta}_{t}-\boldsymbol{\Delta}_{c}^{2}} \\
\lambda_{t}^{\prime}-\frac{\boldsymbol{\Delta}_{c}}{\boldsymbol{\Delta}_{s}} \lambda_{s}^{\prime} \\
\lambda_{a}^{\prime}
\end{array}\right\} \\
\mathbf{F}=\left\{\begin{array}{c}
\boldsymbol{\Delta}_{s} \frac{\boldsymbol{\Delta}_{t} \mathbf{F}_{p}^{s}-\boldsymbol{\Delta}_{c} \mathbf{F}_{p}^{t}}{\boldsymbol{\Delta}_{s} \boldsymbol{\Delta}_{t}-\boldsymbol{\Delta}_{c}^{2}} \\
\mathbf{F}_{p}^{t}-\frac{\boldsymbol{\Delta}_{c}}{\boldsymbol{\Delta}_{s}} \mathbf{F}_{p}^{s} \\
\mathbf{F}_{p}^{a}
\end{array}\right\}, \quad \boldsymbol{\Delta}=\left\{\begin{array}{c}
\boldsymbol{\Delta}_{s} \\
\boldsymbol{\Delta}_{t}-\frac{\boldsymbol{\Delta}_{c}^{2}}{\boldsymbol{\Delta}_{s}} \\
\boldsymbol{\Delta}_{a}
\end{array}\right\},
\end{gathered}
$$

and the linear system reads

$$
\left[\begin{array}{ccc|c}
{\left[\boldsymbol{\Delta}_{s}\right]} & {[\mathbf{0}]} & {[\mathbf{0}]} & \\
{[\mathbf{0}]} & {\left[\boldsymbol{\Delta}_{t}\right]-\frac{\left[\boldsymbol{\Delta}_{c}\right]^{2}}{\left[\boldsymbol{\Delta}_{s}\right]}} & {[\mathbf{0}]} & \mathbf{L}^{\prime} \\
{[\mathbf{0}]} & {[\mathbf{0}]} & {\left[\boldsymbol{\Delta}_{a}\right]} & \\
\hline & { }^{t} \mathbf{L} & & \lambda_{c}
\end{array}\right]\left\{\begin{array}{l}
\mathbf{q}^{s} \\
\mathbf{q}^{t} \\
\frac{\mathbf{q}^{a}}{F^{c}}
\end{array}\right\}=\left\{\begin{array}{c}
\mathbf{F} \\
\overline{u^{c}}
\end{array}\right\}
$$

The idea of the method is to successively replace the last equation of this system by an adequate combination the first ones of the problem as a classical gaussian elimination. This equation initially reads

$$
\left(\sum_{i=1}^{n} L_{i} q_{i}\right)+\lambda_{c} F_{c}=u_{c}
$$

After the first step, this equation is replaced by its difference with $\left(L_{1} / \Delta_{1}\right)$ times the first line and one has

$$
\left(\sum_{i=2}^{n} L_{i} q_{i}\right)+\left\{\lambda_{c}-\frac{L_{1}}{\Delta_{1}} L_{1}^{\prime}\right\} F_{c}=u_{c}-\frac{L_{1}}{\Delta_{1}} F_{1}
$$

This operation is performed $n-1$ so that

$$
\left\{\lambda_{c}-\sum_{i=1}^{n} \frac{L_{i}}{\Delta_{i}} L_{i}^{\prime}\right\} F_{c}=u_{c}-\sum_{i=1}^{n} \frac{L_{i}}{\Delta_{i}} F_{i}
$$


and

$$
F_{c}=\frac{u_{c}-\sum_{i=1}^{n} \frac{L_{i}}{\Delta_{i}} F_{i}}{\lambda_{c}-\sum_{i=1}^{n} \frac{L_{i}}{\Delta_{i}} L_{i}^{\prime}} .
$$

In the case of several $(m)$ Lagrange multiplier, this procedure can be extended and it is needed to solve a linear system of size $m$.

\section{References}

[1] M. A. Biot: Theory of propagation of elastic waves in a fluid-filled-saturated porous solid. Journal of the Acoustical Society of America 28 (1956) 168-191.

[2] M. A. Biot: Mechanics of deformation and acoustic propagation in porous media. Journal of Applied Physics 33(4) (1962) 1482-1498.

[3] J. F. Allard: Propagation of sound in porous media, modelling sound absorbing materials. New York and London, 1993.

[4] D. L. Johnson, J. Koplik, R. Dashen: Theory of dynamic permeability and tortuosity in fluid-saturated porous media. Journal of Fluid Mechanics 176 (1987) 379-402.

[5] Y. Champoux, J. F. Allard: Dynamic tortuosity and bulk modulus in air-saturated porous media. Journal of Applied Physics 70 (1991) 1975-1979.

[6] D. Lafarge, P. Lemarinier, J. F. Allard, V. Tarnow: Dynamic compressibility of air in porous structures at audible frequencies. Journal of the Acoustical Society of America 102 (1997) 1995-2006.

[7] O. Doutres, N. Dauchez, J.-M. Génevaux, G. Lemarquand: On the use of a loudspeaker for measuring the viscoelastic properties of sound absorbing materials. Journal of the Acoustical Society of America (2008).

[8] N. Atalla, R. Panneton, P. Debergue: A mixed displacement-pressure formulation for poroelastic materials. Journal of the Acoustical Society of America 104 (1998) 14441452.

[9] N. Atalla, M. A. Hamdi, R. Panneton: Enhanced weak integral formulation for the mixed (u,p) poroelastic equations. Journal of the Acoustical Society of America 109 (2001) 3065-3068.
[10] A. Bermudes, J. L. Ferrin, A. Pietro: Finite element solution of a new displacement/pressure poroelastic models in acoustics. Computer methods in applied mechanics and engineering 195 (2006) 3509-3516.

[11] O. Dazel, B. Brouard, C. Depollier, S. Griffiths: An alternative Biot's displacement formulation for porous materials. Journal of the Acoustical Society of America 121 (2007) 3509-3516.

[12] S. Rigobert, N. Atalla, F. Sgard: Investigation of the convergence of the mixed displacement-pressure formulation for three-dimensional poroelastic materials using hierarchical elements. Journal of the Acoustical Society of America 114 (2003) 2607.

[13] N. E. Hörlin, M. Nordström, P. Göransson: A 3-d hierarchical fe formulation of biot's equations for elasto-acoustic modelling of porous media. Journal of Sound and Vibration 243 (2001) 633-652.

[14] F. Sgard, N. Atalla, R. Panneton: A modal reduction technique for the finite element formulation of biot's poroelasticity equations in acoustics applied to multilayered structures. 16th International Congress on Acoustics/135th meeting of the Acoustical Society of America, Seattle, WA, USA, 1998, A. S. o. America (ed.), 1183-1184.

[15] O. Dazel, F. Sgard, C.-H. Lamarque, N. Atalla: An extension of complex modes for the resolution of finite-element poroelastic problems. Journal of Sound and Vibration $\mathbf{2 5 3}$ (2002) 421-445.

[16] O. Dazel, F. Sgard, C.-H. Lamarque: Application of generalized complex modes to the calculation of the forced response of three dimensional poroelastic materials. Journal of Sound and Vibration 268 (2003) 555-580.

[17] J.-D. Chazot, J.-L. Guyader: Prediction of transmission loss of double panels with a patch-mobility method. Journal of the Acoustical Society of America 121 (2007) 267-278.

[18] O.-C. Zienkiewicz, R.-L. Taylor: The finite element method: basic formulation and linear problems. Vol. 1. 4th ed. McGraw Hill, London, Great Britain, 1989.

[19] R. Panneton, N. Atalla, F. Charron: A finite-element formulation for the vibroacoustic behavior of double-plate structures with cavity absorption. Canadian aerospace journal 41 (1995) 5-12. 